\title{
The growth rates of digits in the Oppenheim series expansions
}

\author{
by \\ BAO-WeI WANG and Jun Wu (Wuhan)
}

1. Introduction. Let $a_{n}(j)$ and $b_{n}(j), n \geq 1$, be two sequences of positive integer-valued functions of the positive integer $j \geq 1$. The algorithm $0<x \leq 1, x=x_{1}$, and, for any $n \geq 1$ and some positive integers $d_{n}(x)$,

$$
\frac{1}{d_{n}(x)}<x_{n} \leq \frac{1}{d_{n}(x)-1}, \quad x_{n}=\frac{1}{d_{n}(x)}+\frac{a_{n}\left(d_{n}(x)\right)}{b_{n}\left(d_{n}(x)\right)} x_{n+1}
$$

leads to the series expansion

$$
x=\frac{1}{d_{1}(x)}+\sum_{n=1}^{\infty} \frac{a_{1}\left(d_{1}(x)\right) \cdots a_{n}\left(d_{n}(x)\right)}{b_{1}\left(d_{1}(x)\right) \cdots b_{n}\left(d_{n}(x)\right)} \cdot \frac{1}{d_{n+1}(x)},
$$

which is called the Oppenheim series expansion of $x$. Set

$$
h_{n}(j)=\frac{a_{n}(j)}{b_{n}(j)} j(j-1), \quad j \geq 2 .
$$

If $h_{n}(j)$ is integer-valued ( $n \geq 1, j \geq 2$ ), then (2) is termed the restricted Oppenheim series expansion of $x$. Here and in what follows, we always assume $h_{j}$ is integer-valued for all $j \geq 1$.

The algorithm (1) implies

$$
d_{1}(x) \geq 2, \quad d_{n+1}(x) \geq h_{n}\left(d_{n}(x)\right)+1 \quad \text { for any } n \geq 1 .
$$

On the other hand, any integer sequence $\left\{d_{n}, n \geq 1\right\}$ satisfying (4) is an Oppenheim admissible sequence, that is, there exists a unique $x \in(0,1] \mathrm{such}$ that $d_{n}(x)=d_{n}$ for any $n \geq 1$. The representation (2) under (1) is unique.

The representation (2) under (1) was first studied by A. Oppenheim [7] who established its arithmetical properties, including the question of rationality of the expansion. The foundations of the metric theory were laid down by J. Galambos [2]-[4], [6]; see also the monographs of J. Galambos [5],

2000 Mathematics Subject Classification: Primary 11K55; Secondary 28A78, 28A80.

Key words and phrases: Oppenheim series expansions, growth rate, Hausdorff dimension. 
F. Schweiger [8] and W. Vervaat [9]. In particular, concerning the growth of $\left\{d_{n}(x), n \geq 1\right\}$ J. Galambos [5, p. 93] obtained the following interesting result:

Definition 1.1. Let $\beta \geq 1$. We say that the function $h_{n}(j)$ is of order $\beta$ if there are constants $0<C_{1} \leq C_{2}$ such that

$$
C_{1} \leq h_{n}(j) / j^{\beta} \leq C_{2} \quad \text { for all } n \text { and } j .
$$

TheOREM 1.2. Let $h_{n}(j)$ be of order $\beta>1$. Then, for almost all $x \in(0,1]$, the limit

$$
\lim _{n \rightarrow \infty} \beta^{-n} \log d_{n}(x)=G(x)
$$

exists. Its value equals the finite series

$$
G(x)=\beta^{-1}\left\{\log d_{1}(x)+\sum_{n=1}^{\infty} \beta^{-n} \log \left(d_{n+1}(x) d_{n}(x)^{-\beta}\right)\right\} .
$$

From Theorem 1.2, we deduce that when $h_{n}(j)$ is of order $\beta>1$, then for almost all $x \in(0,1]$,

$$
\lim _{j \rightarrow \infty} \frac{\log d_{j+1}(x)}{\log h_{j}\left(d_{j}(x)\right)}=1 .
$$

Hence a natural problem is to discuss the size of the sets with different growth rates of $\left\{d_{n}(x), n \geq 1\right\}$. More precisely, for any $\alpha \geq 1$, let

$$
B_{\alpha}=\left\{x \in(0,1]: \lim _{j \rightarrow \infty} \frac{\log d_{j+1}(x)}{\log h_{j}\left(d_{j}(x)\right)}=\alpha\right\} .
$$

What is the size of $B_{\alpha}$ ? In this paper, we calculate its Hausdorff dimension. The situation here is quite complicated and we carefully construct a Cantor set $E \subset B_{\alpha}$ such that the Hausdorff dimension of $E$ approximates that of $B_{\alpha}$. Some other exceptional sets associated with the Oppenheim series expansion were discussed in [10]-[12].

We use $|\cdot|$ to denote the diameter of a subset of $(0,1], \operatorname{dim}_{H}$ to denote the Hausdorff dimension and $\mathrm{cl}$ for the closure of a subset of $(0,1]$.

2. Hausdorff dimension of $B_{\alpha}$. In this section, we give the main result of this paper.

We start with the mass distribution principle (see [1, Proposition 2.3]) that will be used later.

Lemma 2.1. Let $E \subset(0,1]$ be a Borel set, and $\mu$ a measure with $\mu(E)>0$. If for any $x \in E$,

$$
\liminf _{r \rightarrow 0} \frac{\log \mu(B(x, r))}{\log r} \geq s
$$

where $B(x, r)$ denotes the open ball with center at $x$ and radius $r$, then $\operatorname{dim}_{\mathrm{H}} E \geq s$. 
The following result is proved in [10].

Lemma 2.2. Suppose $h_{n}(j) \geq j-1$ for any $n \geq 1$ and $j \geq 2$. Then for any $m \geq 3$, the set

$$
C_{m}=\left\{x \in(0,1]: 1<\frac{d_{j}(x)}{h_{j-1}\left(d_{j-1}(x)\right)} \leq m \text { for any } j \geq 2\right\}
$$

has Hausdorff dimension 1.

Now we state our main result.

TheOREM 2.3. Let $h_{n}(j)$ be of order $\beta \geq 1$ and $h_{n}(j) \geq j-1$ for any $n \geq 1$ and $j \geq 2$. Then for any $\alpha \geq 1$,

$$
\operatorname{dim}_{\mathrm{H}} B_{\alpha}=\frac{1}{(\alpha-1) \beta+1} .
$$

Proof. Let

$$
D=\left\{x \in(0,1]: \limsup _{j \rightarrow \infty} d_{j}(x)<\infty\right\}
$$

By (4) and the assumption $h_{n}(j) \geq j-1$ for any $n \geq 1$ and $j \geq 2$, we have $d_{j+1}(x) \geq h_{j}\left(d_{j}(x)\right)+1 \geq d_{j}(x)$. Thus for any $x \in D, d_{j+1}(x)=d_{j}(x)$ ultimately, and therefore

$$
D \subset \bigcup_{k=1}^{\infty} \bigcup_{t=2}^{\infty}\left\{x \in(0,1]: d_{j}(x)=t \text { for any } j \geq k\right\},
$$

which implies $D$ is countable.

If $\alpha=1$, then for any $x \in C_{m} \backslash D$, where $C_{m}$ is defined in Lemma 2.2, we have

$$
\lim _{j \rightarrow \infty} \frac{\log d_{j+1}(x)}{\log h_{j}(x)}=1 .
$$

Thus $C_{m} \backslash D \subset B_{1}$. By Lemma 2.2 , we have $\operatorname{dim}_{\mathrm{H}} B_{1}=1$.

In the following, we always assume that $\alpha>1$. We divide the proof into two parts.

PART I: Upper bound. Let $\varepsilon<\min \{\alpha-1 / \beta, \alpha-1\}$. For any $x \in B_{\alpha} \backslash D$, from the definition of $B_{\alpha}$, there exists $j_{0}$ such that for any $j \geq j_{0}$,

$$
h_{j}^{\alpha-\varepsilon}\left(d_{j}(x)\right)<d_{j+1}(x)<h_{j}^{\alpha+\varepsilon}\left(d_{j}(x)\right) .
$$

Thus

where

$$
B_{\alpha} \backslash D \subset \bigcup_{j_{0}=1}^{\infty} B_{\alpha}\left(\varepsilon, j_{0}\right),
$$

$$
B_{\alpha}\left(\varepsilon, j_{0}\right)=\left\{x \in(0,1]: h_{j}^{\alpha-\varepsilon}\left(d_{j}(x)\right)<d_{j+1}(x)<h_{j}^{\alpha+\varepsilon}\left(d_{j}(x)\right) \text { for } j \geq j_{0}\right\} .
$$

Fix $j_{0} \geq 1$; we now estimate $\operatorname{dim}_{\mathrm{H}} B_{\alpha}\left(\varepsilon, j_{0}\right)$ from above. 
Since $h_{j}(d)$ is of order $\beta$, for any

$$
0<\eta<\min \left(1, \frac{\beta(\alpha-1-\varepsilon)}{\alpha+1-\varepsilon}, \beta-\frac{1}{\alpha-\varepsilon}\right)
$$

there exists $d_{0}>3^{1 / 2 \beta \varepsilon}$ such that for any $d \geq d_{0}$,

$$
d^{\beta-\eta}<h_{j}(d)<d^{\beta+\eta} .
$$

For any $x \in B_{\alpha}\left(\varepsilon, j_{0}\right) \backslash D$, since $d_{j}(x) \rightarrow \infty$ as $j \rightarrow \infty$, there exists $j_{1}$ such that $d_{j}(x) \geq d_{0}$ for any $j \geq j_{1}$. Thus we have

$$
B_{\alpha}\left(\varepsilon, j_{0}\right) \backslash D \subset \bigcup_{j_{1}=1}^{\infty} B_{\alpha}\left(\varepsilon, j_{0}, \eta, j_{1}\right),
$$

where

$$
\begin{aligned}
B_{\alpha}\left(\varepsilon, j_{0}, \eta, j_{1}\right)=\left\{x \in(0,1]: h_{j}^{\alpha-\varepsilon}\left(d_{j}(x)\right)\right. & <d_{j+1}(x)<h_{j}^{\alpha+\varepsilon}\left(d_{j}(x)\right) \text { for } \\
& \left.j \geq j_{0} \text { and } d_{j}(x) \geq d_{0} \text { for } j \geq j_{1}\right\} .
\end{aligned}
$$

For any $j_{1} \geq 1$, let $j_{2}=\max \left\{j_{1}, j_{0}\right\}$. Then $B_{\alpha}\left(\varepsilon, j_{0}, \eta, j_{1}\right)$ is contained in

$$
\begin{aligned}
& \left\{x \in(0,1]: h_{j}^{\alpha-\varepsilon}\left(d_{j}(x)\right)<d_{j+1}(x)<h_{j}^{\alpha+\varepsilon}\left(d_{j}(x)\right)\right. \\
& \left.\quad \text { and } d_{j}(x) \geq d_{0} \text { for any } j \geq j_{2}\right\} \\
& =\bigcup_{d_{1}, \ldots, d_{j_{2}-1}}\left\{x \in(0,1]: d_{1}(x)=d_{1}, \ldots, d_{j_{2}-1}(x)=d_{j_{2}-1}, d_{j_{2}}(x) \geq d_{0},\right. \\
& \left.\quad \text { and } h_{j}^{\alpha-\varepsilon}\left(d_{j}(x)\right)<d_{j+1}(x)<h_{j}^{\alpha+\varepsilon}\left(d_{j}(x)\right) \text { for any } j \geq j_{2}\right\} \\
& \subset \bigcup_{d_{1}, \ldots, d_{j_{2}-1}, d_{j_{2}} \geq d_{0}}\left\{x \in(0,1]: d_{1}(x)=d_{1}, \ldots, d_{j_{2}}(x)=d_{j_{2}},\right. \\
& \left.\qquad d_{j}^{(\beta-\eta)(\alpha-\varepsilon)}(x)<d_{j+1}(x)<d_{j}^{(\beta+\eta)(\alpha+\varepsilon)}(x) \text { for any } j \geq j_{2}\right\},
\end{aligned}
$$

where the union is over all $d_{1}, \ldots, d_{j_{2}-1}, d_{j_{2}}$ such that $d_{1} \geq 2, d_{j_{2}} \geq d_{0}$ and $d_{j+1} \geq h_{j}\left(d_{j}\right)+1$ for any $1 \leq j \leq j_{2}-1$.

For any $\bar{d}=\left(d_{1}, \ldots, d_{j_{2}-1}, d_{j_{2}}\right)$ satisfying the above conditions, let

$$
\begin{aligned}
& \Gamma\left(\varepsilon, j_{0}, \eta, j_{2}, \bar{d}\right)=\left\{x \in(0,1]: d_{1}(x)=d_{1}, \ldots, d_{j_{2}}(x)=d_{j_{2}},\right. \\
& \left.\left[d_{j}^{(\beta-\eta)(\alpha-\varepsilon)}(x)\right]<d_{j+1}(x) \leq\left[d_{j}^{(\beta+\eta)(\alpha+\varepsilon)}(x)\right]+1 \text { for any } j \geq j_{2}\right\} .
\end{aligned}
$$

By the $\sigma$-stability of Hausdorff dimension (notice that $D$ is countable), in order to get an upper bound of $\operatorname{dim}_{\mathrm{H}} B_{\alpha}\left(\varepsilon, j_{0}\right)$, it suffices to give an upper bound of $\operatorname{dim}_{\mathrm{H}} \Gamma\left(\varepsilon, j_{0}, \eta, j_{2}, \bar{d}\right)$ for any $j_{2} \geq j_{0}$ and any $\bar{d}=\left(d_{1}, \ldots, d_{j_{2}-1}, d_{j_{2}}\right)$ as above. 
Now we introduce a kind of symbolic space defined as follows. For any $k \geq j_{2}+1$, let

$$
\begin{aligned}
D_{k}=\{\sigma= & \left(\sigma_{1}, \ldots, \sigma_{k}\right) \in \mathbb{N}^{k}: \sigma_{1}=d_{1}, \ldots, \sigma_{j_{2}}=d_{j_{2}}, \\
& {\left.\left[\sigma_{j}^{(\beta-\eta)(\alpha-\varepsilon)}\right]<\sigma_{j+1} \leq\left[\sigma_{j}^{(\beta+\eta)(\alpha+\varepsilon)}\right]+1 \text { for } j_{2} \leq j<k\right\}, }
\end{aligned}
$$

and define

$$
D^{*}=\bigcup_{k=j_{2}+1}^{\infty} D_{k} \text {. }
$$

For any $k \geq j_{2}+1$ and $\sigma=\left(\sigma_{1}, \ldots, \sigma_{k}\right) \in D_{k}$, let $J_{\sigma}$ and $I_{\sigma}$ denote the following closed subintervals of $(0,1]$ :

$$
\begin{aligned}
& J_{\sigma}=\underset{\left[\sigma_{k}^{(\beta-\eta)(\alpha-\varepsilon)}\right]<d \leq\left[\sigma_{k}^{(\beta+\eta)(\alpha+\varepsilon)}\right]+1}{\bigcup} \operatorname{cl}\left\{x \in(0,1]: d_{1}(x)=\sigma_{1}, \ldots, d_{k}(x)=\sigma_{k},\right. \\
& I_{\sigma}=\operatorname{cl}\left\{x \in(0,1]: d_{1}(x)=\sigma_{1}, \ldots, d_{k}(x)=\sigma_{k}\right\} .
\end{aligned}
$$

By the restriction on $\eta$, we know that $J_{\sigma} \neq \emptyset$, since, for any $j \geq j_{2}$,

$$
\sigma_{j+1}>\sigma_{j}^{(\beta-\eta)(\alpha-\varepsilon)}>\sigma_{j}>\cdots>\sigma_{j_{2}} \geq d_{0} .
$$

It follows that

$$
\sigma_{j+1}>\sigma_{j}^{(\beta-\eta)(\alpha-\varepsilon)}>h_{j}^{\frac{(\beta-\eta)(\alpha-\varepsilon)}{\beta+\eta}}\left(\sigma_{j}\right) \geq h_{j}\left(\sigma_{j}\right) .
$$

Moreover, we know that $\sigma_{k} \geq d_{0}$ for any $k \geq j_{2}+1$, which yields

$$
\sigma_{k}^{2(\alpha \eta+\beta \varepsilon)} \geq d_{0}^{2(\alpha \eta+\beta \varepsilon)} \geq 3^{\frac{2(\alpha \eta+\beta \varepsilon)}{2 \beta \varepsilon}} \geq 3 .
$$

Each $J_{\sigma}$ is called an interval of $k$ th order. Finally, define

$$
E=\bigcap_{k=j_{2}+1}^{\infty} \bigcup_{\sigma \in D_{k}} J_{\sigma} .
$$

It is obvious that

$$
\Gamma\left(\varepsilon, j_{0}, \eta, j_{2}, \bar{d}\right)=E .
$$

From the proof of Theorem 6.1 in [5], we have, for any $k \geq j_{2}+1$ and $\sigma \in D_{k}$,

$$
\begin{aligned}
\left|I_{\sigma}\right| & =\frac{a_{1}\left(\sigma_{1}\right)}{b_{1}\left(\sigma_{1}\right)} \cdot \frac{a_{2}\left(\sigma_{2}\right)}{b_{2}\left(\sigma_{2}\right)} \cdots \frac{a_{k-1}\left(\sigma_{k-1}\right)}{b_{k-1}\left(\sigma_{k-1}\right)} \cdot \frac{1}{\left(\sigma_{k}-1\right) \sigma_{k}} \\
\left|J_{\sigma}\right| & =\sum_{\left[\sigma_{k}^{(\beta-\eta)(\alpha-\varepsilon)}\right]<d \leq\left[\sigma_{k}^{(\beta+\eta)(\alpha+\varepsilon)}\right]+1} \frac{a_{1}\left(\sigma_{1}\right)}{b_{1}\left(\sigma_{1}\right)} \cdots \frac{a_{k}\left(\sigma_{k}\right)}{b_{k}\left(\sigma_{k}\right)} \cdot \frac{1}{(d-1) d} \\
& =\frac{a_{1}\left(\sigma_{1}\right)}{b_{1}\left(\sigma_{1}\right)} \cdots \frac{a_{k}\left(\sigma_{k}\right)}{b_{k}\left(\sigma_{k}\right)}\left(\frac{1}{\left[\sigma_{k}^{(\beta-\eta)(\alpha-\varepsilon)}\right]}-\frac{1}{\left[\sigma_{k}^{(\beta+\eta)(\alpha+\varepsilon)}\right]+1}\right)
\end{aligned}
$$


For any

$$
s>\frac{(\alpha+\varepsilon)(\beta+\eta)}{(\alpha-\varepsilon)(\beta-\eta)[2+(\alpha-\varepsilon)(\beta-\eta)-(\beta+\eta)]-(\alpha+\varepsilon)(\beta+\eta)},
$$

by (9) and (11), we have

$$
\begin{aligned}
\mathbf{H}^{s}(E) & \leq \liminf _{k \rightarrow \infty} \sum_{\sigma \in D_{k}}\left|J_{\sigma}\right|^{s} \\
= & \liminf _{k \rightarrow \infty} \sum_{\sigma \in D_{k}}\left(\frac{a_{1}\left(\sigma_{1}\right)}{b_{1}\left(\sigma_{1}\right)} \cdots \frac{a_{k}\left(\sigma_{k}\right)}{b_{k}\left(\sigma_{k}\right)}\left(\frac{1}{\left[\sigma_{k}^{(\beta-\eta)(\alpha-\varepsilon)}\right]}-\frac{1}{\left[\sigma_{k}^{(\beta+\eta)(\alpha+\varepsilon)}\right]+1}\right)^{s}\right. \\
= & \liminf _{k \rightarrow \infty} \sum_{\sigma \in D_{k-1}}\left|J_{\sigma}\right|^{s}\left(\frac{h_{k}\left(\sigma_{k}\right)}{\sigma_{k}\left(\sigma_{k}-1\right)}\right)^{s} \\
& \times\left(\frac{1}{\left[\sigma_{k}^{(\alpha-\varepsilon)(\beta-\eta)}\right]}-\frac{1}{\left[\sigma_{k-1}^{(\alpha-\varepsilon)(\beta-\eta)}\right]<\sigma_{k} \leq\left[\sigma_{k-1}^{(\alpha+\varepsilon)(\beta+\eta)}\right]+1}\right)^{s} \\
& \times\left(\frac{1}{\left[\sigma_{k-1}^{(\alpha-\varepsilon)(\beta-\eta)(\beta+\eta)}\right]+1}\right)^{-s}
\end{aligned}
$$$$
\leq \liminf _{k \rightarrow \infty} \sum_{\sigma \in D_{k-1}}\left|J_{\sigma}\right|^{s} \sum_{\left[\sigma_{k-1}^{(\alpha-\varepsilon)(\beta-\eta)}\right]<\sigma_{k} \leq\left[\sigma_{k-1}^{(\alpha+\varepsilon)(\beta+\eta)}\right]+1}\left(\frac{\sigma_{k}^{\beta+\eta}}{\sigma_{k}^{2}} \cdot \frac{\sigma_{k}}{\sigma_{k}-1}\right)^{s}
$$$$
\times\left(\frac{1}{\left[\sigma_{k}^{(\alpha-\varepsilon)(\beta-\eta)}\right]} \cdot \frac{\sigma_{k-1}^{(\alpha+\varepsilon)(\beta+\eta)}+1}{\sigma_{k-1}^{2(\alpha \eta+\beta \varepsilon)}-1}\right)^{s}
$$

$$
\begin{aligned}
& \leq \liminf _{k \rightarrow \infty} \sum_{\sigma \in D_{k-1}}\left|J_{\sigma}\right|^{s} \sum_{\left[\sigma_{k-1}^{(\alpha-\varepsilon)(\beta-\eta)}\right]<\sigma_{k} \leq\left[\sigma_{k-1}^{(\alpha+\varepsilon)(\beta+\eta)}\right]+1}\left(\frac{\sigma_{k}^{\beta+\eta}}{\sigma_{k}^{2}} \cdot \frac{\sigma_{k}}{\sigma_{k}-1}\right)^{s} \\
& \quad \times\left(\frac{1}{\sigma_{k}^{(\alpha-\varepsilon)(\beta-\eta)}-1} \sigma_{k-1}^{(\alpha+\varepsilon)(\beta+\eta)}\right)^{s} .
\end{aligned}
$$

For any $k \geq j_{2}+1$, let

$$
a_{k}:=\frac{\sigma_{k}}{\sigma_{k}-1} \cdot \frac{\sigma_{k}^{(\alpha-\varepsilon)(\beta-\eta)}}{\sigma_{k}^{(\alpha-\varepsilon)(\beta-\eta)}-1}
$$

Since $\log (1+x)<x$ for any $x>0$, and $(\alpha-\varepsilon)(\beta-\eta)>1$, we have

$$
\begin{aligned}
\log a_{k} & <\frac{1}{\sigma_{k}-1}+\frac{1}{\sigma_{k}^{(\alpha-\varepsilon)(\beta-\eta)}-1}<\frac{4}{\sigma_{k}}<\frac{4}{d_{0}^{((\alpha-\varepsilon)(\beta-\eta))^{k-j_{2}}}} \\
& <\frac{4}{d_{0}^{((\alpha-\varepsilon)(\beta-\eta)-1)\left(k-j_{2}\right)}}=: \frac{4}{r^{k-j_{2}}} \quad(r>1) .
\end{aligned}
$$


Thus

$$
\begin{aligned}
\mathbf{H}^{s}(E) \leq & \liminf _{k \rightarrow \infty} \sum_{\sigma \in D_{k-1}}\left|J_{\sigma}\right|^{s} \sum_{\left[\sigma_{k-1}^{(\alpha-\varepsilon)(\beta-\eta)}\right]<\sigma_{k} \leq\left[\sigma_{k-1}^{(\alpha+\varepsilon)(\beta+\eta)}\right]+1}\left(e^{\left.4 / r^{k-j_{2}}\right)^{s}}\right. \\
& \times\left(\frac{\sigma_{k}^{\beta+\eta}}{\sigma_{k}^{2}} \cdot \frac{1}{\sigma_{k}^{(\alpha-\varepsilon)(\beta-\eta)}} \sigma_{k-1}^{(\alpha+\varepsilon)(\beta+\eta)}\right)^{s} \\
\leq & \liminf _{k \rightarrow \infty} \sum_{\sigma \in D_{k-1}}\left|J_{\sigma}\right|^{s} e^{4 s / r^{k-j_{2}}} \sigma_{k-1}^{(\alpha+\varepsilon)(\beta+\eta)} \\
& \times\left(\frac{1}{\sigma_{k-1}^{(\alpha-\varepsilon)(\beta-\eta)(2+(\alpha-\varepsilon)(\beta-\eta)-(\beta+\eta))}} \sigma_{k-1}^{(\alpha+\varepsilon)(\beta+\eta)}\right)^{s} .
\end{aligned}
$$

By (12) we have

Thus

$$
\begin{aligned}
\mathbf{H}^{s}(E) & \leq \liminf _{k \rightarrow \infty} \sum_{\sigma \in D_{k-1}}\left|J_{\sigma}\right|^{s} e^{4 s / r^{k-j_{2}}} \\
& \leq \cdots \leq \lim _{k \rightarrow \infty} \prod_{i=j_{2}+1}^{k} e^{4 s / r^{i-j_{2}}} \sum_{\sigma \in D_{j_{2}+1}}\left|J_{\sigma}\right|^{s}<\infty
\end{aligned}
$$

$$
\operatorname{dim}_{\mathrm{H}} E \leq \frac{(\alpha+\varepsilon)(\beta+\eta)}{(\alpha-\varepsilon)(\beta-\eta)[2+(\alpha-\varepsilon)(\beta-\eta)-(\beta+\eta)]-(\alpha+\varepsilon)(\beta+\eta)} .
$$

By the $\sigma$-stability of Hausdorff dimension, we have, for any $j_{1} \geq 1$, $\operatorname{dim}_{\mathrm{H}} B_{\alpha}\left(\varepsilon, j_{0}, \eta, j_{1}\right) \leq \sup _{d_{1}, \ldots, d_{j_{2}-1}, d_{j_{2}} \geq d_{0}} \operatorname{dim}_{\mathrm{H}} \Gamma\left(\varepsilon, j_{0}, \eta, j_{2}, \bar{d}\right)$

$$
\leq \frac{(\alpha+\varepsilon)(\beta+\eta)}{(\alpha-\varepsilon)(\beta-\eta)[2+(\alpha-\varepsilon)(\beta-\eta)-(\beta+\eta)]-(\alpha+\varepsilon)(\beta+\eta)} .
$$

This implies

$$
\begin{aligned}
\operatorname{dim}_{\mathrm{H}} & B_{\alpha}\left(\varepsilon, j_{0}\right) \\
\leq & \leq \frac{(\alpha+\varepsilon)(\beta+\eta)}{(\alpha-\varepsilon)(\beta-\eta)[2+(\alpha-\varepsilon)(\beta-\eta)-(\beta+\eta)]-(\alpha+\varepsilon)(\beta+\eta)} .
\end{aligned}
$$

Since $\eta$ is arbitrary, we get

$$
\operatorname{dim}_{\mathrm{H}} B_{\alpha}\left(\varepsilon, j_{0}\right) \leq \frac{(\alpha+\varepsilon) \beta}{(\alpha-\varepsilon) \beta[2+(\alpha-\varepsilon) \beta-\beta]-(\alpha+\varepsilon) \beta} .
$$

The $\sigma$-stability of Hausdorff dimension yields

$$
\operatorname{dim}_{\mathrm{H}} B_{\alpha} \leq \frac{(\alpha+\varepsilon) \beta}{(\alpha-\varepsilon) \beta[2+(\alpha-\varepsilon) \beta-\beta]-(\alpha+\varepsilon) \beta} .
$$

Since $\varepsilon$ is arbitrary, we have

$$
\operatorname{dim}_{\mathrm{H}} B_{\alpha} \leq \frac{1}{(\alpha-1) \beta+1}
$$

This completes the proof of Part I. 
PART II: Lower bound. Since $h_{j}(d)$ is of order $\beta$, there exists $c>2$ such that for any $j \geq 1$ and $d \geq 2$,

$$
c^{-1} d^{\beta} \leq h_{j}(d) \leq c d^{\beta} .
$$

Since $\alpha>1$, there exist $d_{0}>4$ and $K_{0}$ such that for any $d \geq d_{0}, j \geq K_{0}$, we have

$$
c^{-\alpha} d^{\beta \alpha} \geq c d^{\beta}+1, \quad(d-1)^{\alpha}-1 \geq(d-1)^{(j+1) / j} .
$$

This implies, for any $d \geq d_{0}$,

$$
h_{j}^{\alpha}(d) \geq\left(c^{-1} d^{\beta}\right)^{\alpha} \geq c d^{\beta}+1 \geq h_{j}(d)+1 .
$$

Let $j_{0} \geq \max \left(\beta / 3, K_{0}\right), j_{0} \in \mathbb{N}$. Choose an integer sequence $d_{1}, \ldots, d_{j_{0}}$ satisfying $d_{1} \geq 2, d_{j+1} \geq h_{j}\left(d_{j}\right)+1,1 \leq j \leq j_{0}-1$, and

$$
d_{j_{0}}>\max \left\{d_{0}, c^{3} 2^{\beta+2}+1\right\}, \quad\left(d_{j_{0}}-1\right)^{\alpha}\left[\left(d_{j_{0}}-1\right)^{2 / j_{0}}-1\right]>2 .
$$

Define

$$
\begin{aligned}
B_{\alpha}^{(1)}\left(j_{0}\right)=\{x \in(0,1]: & d_{1}(x)=d_{1}, \ldots, d_{j_{0}}(x)=d_{j_{0}}, \\
& \left.h_{j}^{\alpha}\left(d_{j}(x)\right)<d_{j+1}(x) \leq h_{j}^{\alpha+2 / j}\left(d_{j}(x)\right) \text { for } j \geq j_{0}\right\} .
\end{aligned}
$$

Then $B_{\alpha}^{(1)}\left(j_{0}\right) \neq \emptyset$. In fact, by $(17)$ and $h_{j}(d) \geq d-1$ for any $j$ and $d$, we have

$$
\begin{aligned}
h_{j_{0}}^{\alpha+2 / j_{0}}\left(d_{j_{0}}\right)-h_{j_{0}}^{\alpha}\left(d_{j_{0}}\right) & =h_{j_{0}}^{\alpha}\left(d_{j_{0}}\right)\left(h_{j_{0}}^{2 / j_{0}}\left(d_{j_{0}}\right)-1\right) \\
& \geq\left(d_{j_{0}}-1\right)^{\alpha}\left[\left(d_{j_{0}}-1\right)^{2 / j_{0}}-1\right]>2 .
\end{aligned}
$$

Then there exists $d_{j_{0}+1} \in \mathbb{N}$ satisfying $h_{j_{0}}^{\alpha}\left(d_{j_{0}}\right)<d_{j_{0}+1} \leq h_{j_{0}}^{\alpha+2 / j_{0}}\left(d_{j_{0}}\right)$, and, by (16),

$$
d_{j_{0}+1} \geq h_{j_{0}}^{\alpha}\left(d_{j_{0}}\right) \geq h_{j_{0}}\left(d_{j_{0}}\right)+1 .
$$

Suppose by induction there exist $d_{j_{0}+1}, d_{j_{0}+2}, \ldots, d_{j} \in \mathbb{N}$ satisfying

$$
h_{k-1}^{\alpha}\left(d_{k-1}\right)<d_{k} \leq h_{k-1}^{\alpha+2 /(k-1)}\left(d_{k-1}\right), \quad j_{0}+1 \leq k \leq j,
$$

and

$$
d_{k} \geq h_{k-1}\left(d_{k-1}\right)+1, \quad j_{0}+1 \leq k \leq j .
$$

By (15) and (17), we have

$$
\begin{aligned}
h_{j}^{\alpha+2 / j}\left(d_{j}\right)-h_{j}^{\alpha}\left(d_{j}\right) & =h_{j}^{\alpha}\left(d_{j}\right)\left(h_{j}^{2 / j}\left(d_{j}\right)-1\right) \\
& \geq\left(d_{j}-1\right)^{\alpha}\left(\left(d_{j}-1\right)^{2 / j}-1\right) \\
& \geq\left(h_{j-1}^{\alpha}\left(d_{j-1}\right)-1\right)^{\alpha}\left[\left(h_{j-1}^{\alpha}\left(d_{j-1}\right)-1\right)^{2 / j}-1\right] \\
& \geq\left(\left(d_{j-1}-1\right)^{\alpha}-1\right)^{\alpha}\left[\left(\left(d_{j-1}-1\right)^{\alpha}-1\right)^{2 / j}-1\right] \\
& \geq\left(d_{j-1}-1\right)^{\frac{j}{j-1} \alpha}\left[\left(d_{j-1}-1\right)^{\frac{2}{j} \cdot \frac{j}{j-1}}-1\right]
\end{aligned}
$$




$$
\begin{aligned}
& \geq\left(d_{j-1}-1\right)^{\alpha}\left[\left(d_{j-1}-1\right)^{2 /(j-1)}-1\right] \\
& \geq \cdots \geq\left(d_{j_{0}}-1\right)^{\alpha}\left[\left(d_{j_{0}}-1\right)^{2 / j_{0}}-1\right]>2 .
\end{aligned}
$$

Thus there exists $d_{j+1} \in \mathbb{N}$ satisfying $h_{j}^{\alpha}\left(d_{j}\right)<d_{j+1} \leq h_{j}^{\alpha+2 / j}\left(d_{j}\right)$, and, by (16),

$$
d_{j+1}>h_{j}^{\alpha}\left(d_{j}\right) \geq h_{j}\left(d_{j}\right)+1 .
$$

Therefore $B_{\alpha}^{(1)}\left(j_{0}\right) \neq \emptyset$. From (16), it is clear that for any $x \in B_{\alpha}^{(1)}\left(j_{0}\right)$, $d_{j}(x) \rightarrow \infty$ as $j \rightarrow \infty$ and $B_{\alpha}^{(1)}\left(j_{0}\right) \subset B_{\alpha}$.

Fix $x_{0} \in B_{\alpha}^{(1)}\left(j_{0}\right)$ and choose any $t$ satisfying

$$
t>\frac{\alpha}{\alpha \beta-1}+\frac{2 \alpha}{\beta(\alpha \beta-1)} .
$$

Since $d_{j}\left(x_{0}\right) \rightarrow \infty$ as $j \rightarrow \infty$, there exists $j_{1} \geq j_{0}$ such that for any $j \geq j_{1}$,

$$
d_{j}\left(x_{0}\right) \geq \max \left(9^{3 / 2 \alpha}, c\right)^{t} .
$$

Now

$$
t>\frac{\alpha}{\alpha \beta-1}+\frac{2 \alpha}{\beta(\alpha \beta-1)}
$$

implies that there exists $j_{2} \geq j_{1}$ such that for any $j \geq j_{2}$,

$$
\left(t-\frac{\alpha}{\alpha \beta-1}\right)(\alpha \beta-1)\left(j-j_{1}\right)>\frac{2(\alpha j+1)}{\beta}-\frac{\alpha}{\alpha \beta-1} .
$$

Define

$$
\begin{gathered}
B_{\alpha}^{(2)}\left(j_{1}\right)=\left\{x \in(0,1]: d_{1}(x)=d_{1}\left(x_{0}\right), \ldots, d_{j_{1}}(x)=d_{j_{1}}\left(x_{0}\right),\right. \\
\left.h_{j}^{\alpha}\left(d_{j}(x)\right)<d_{j+1}(x) \leq h_{j}^{\alpha+2 / j}\left(d_{j}(x)\right) \text { for any } j \geq j_{1}\right\}, \\
B_{\alpha}^{(3)}\left(j_{2}\right)=\left\{x \in(0,1]: d_{1}(x)=d_{1}\left(x_{0}\right), \ldots, d_{j_{2}}(x)=d_{j_{2}}\left(x_{0}\right),\right. \\
\left.h_{j}^{\alpha}\left(d_{j}(x)\right)<d_{j+1}(x) \leq h_{j}^{\alpha+2 / j}\left(d_{j}(x)\right) \text { for any } j \geq j_{2}\right\} .
\end{gathered}
$$

For any $x \in B_{\alpha}^{(3)}\left(j_{2}\right)$ and $j \geq j_{2}$, by (19), we have

$$
\begin{aligned}
d_{j}(x) & >h_{j-1}^{\alpha}\left(d_{j-1}(x)\right) \geq\left(c^{-1} d_{j-1}^{\beta}(x)\right)^{\alpha}=\left(c^{-1}\right)^{\alpha} d_{j-1}^{\alpha \beta}(x) \\
& >\left(c^{-1}\right)^{\alpha} h_{j-2}^{\alpha^{2} \beta}\left(d_{j-2}(x)\right) \geq\left(c^{-1}\right)^{\alpha}\left(c^{-1} d_{j-2}^{\beta}(x)\right)^{\alpha^{2} \beta} \\
& =\left(c^{-1}\right)^{\alpha}\left(c^{-1}\right)^{\alpha^{2} \beta} d_{j-2}^{(\alpha \beta)^{2}}(x) \geq \cdots \\
& \geq\left(c^{-1}\right)^{\alpha} \cdots\left(c^{-1}\right)^{(\alpha \beta)^{j-j_{1}-1} \alpha} d_{j_{1}}^{(\alpha \beta)^{j-j_{1}}}(x) \\
& \geq\left(c^{-1}\right)^{\alpha \frac{(\alpha \beta)^{j-j_{1}-1}}{\alpha \beta-1}} \cdot c^{t(\alpha \beta)^{j-j_{1}}} \\
& \geq c^{\left(t-\frac{\alpha}{\alpha \beta-1}\right)(\alpha \beta-1)\left(j-j_{1}\right)+\frac{\alpha}{\alpha \beta-1}}>c^{\frac{2(\alpha j+1)}{\beta}} .
\end{aligned}
$$


On the other hand, if $c>9^{3 / 2 \alpha}$, from (21), we have

$$
d_{j}(x) \geq 9^{\frac{3}{2 \alpha} \cdot \frac{2(\alpha j+1)}{\beta}} \geq 9^{3 j / \beta},
$$

while if $c \leq 9^{3 / 2 \alpha}$, then $c^{-1} \geq 9^{-3 / 2 \alpha}$, and from (19), in the same way as in the proof of (21), we have

$$
\begin{aligned}
d_{j}(x) & >\left(c^{-1}\right)^{\alpha} \cdots\left(c^{-1}\right)^{(\alpha \beta)^{j-j_{1}-1} \alpha} d_{j_{1}}^{(\alpha \beta)^{j-j_{1}}}(x) \\
& \geq\left(9^{-3 / 2 \alpha}\right)^{\alpha} \cdots\left(9^{-3 / 2 \alpha}\right)^{(\alpha \beta)^{j-j_{1}-1} \alpha} \cdot\left(9^{3 / 2 \alpha}\right)^{t(\alpha \beta)^{j-j_{1}}} \geq 9^{3 j / \beta} .
\end{aligned}
$$

So for any $x \in B_{\alpha}^{(3)}\left(j_{2}\right)$ and $j \geq j_{2}$, we have

$$
d_{j}(x)>9^{3 j / \beta} .
$$

Let

$$
\begin{aligned}
& B_{\alpha}^{(4)}\left(j_{2}\right)=\left\{x \in(0,1]: d_{1}(x)=d_{1}\left(x_{0}\right), \ldots, d_{j_{2}}(x)=d_{j_{2}}\left(x_{0}\right),\right.\left.\left(c d_{j}^{\beta}(x)\right)^{\alpha}<d_{j+1}(x) \leq\left(c^{-1} d_{j}^{\beta}(x)\right)^{\alpha+2 / j} \text { for any } j \geq j_{2}\right\}, \\
& B_{\alpha}^{(5)}\left(j_{2}\right)=\left\{x \in(0,1]: d_{1}(x)=d_{1}\left(x_{0}\right), \ldots, d_{j_{2}}(x)=d_{j_{2}}\left(x_{0}\right),\right. \\
&\left.d_{j}^{\left(\beta+\frac{\beta}{2(\alpha j+1)}\right) \alpha}(x)<d_{j+1}(x) \leq d_{j}^{\left(\beta-\frac{\beta}{2(\alpha j+1)}\right)\left(\alpha+\frac{2}{j}\right)}(x) \text { for } j \geq j_{2}\right\} .
\end{aligned}
$$

By (21), we have

$$
B_{\alpha}^{(5)}\left(j_{2}\right) \subset B_{\alpha}^{(4)}\left(j_{2}\right) \subset B_{\alpha}^{(3)}\left(j_{2}\right) \subset B_{\alpha}^{(2)}\left(j_{1}\right) \subset B_{\alpha}^{(1)}\left(j_{0}\right) \subset B_{\alpha} .
$$

For any $j \geq j_{2}$, write

$$
s_{j}=\alpha\left(\beta+\frac{\beta}{2(\alpha j+1)}\right), \quad t_{j}=\left(\beta-\frac{\beta}{2(\alpha j+1)}\right)\left(\alpha+\frac{2}{j}\right) .
$$

Then for any $j \geq j_{2}$,

$$
t_{j}=s_{j}+\frac{\beta}{j}
$$

For any $j \geq j_{2}+1$, define

$$
L_{j}=\left(1+\frac{1}{j^{2}}\right) d_{j_{2}}^{\prod_{i=j_{2}}^{j-1} \frac{s_{i}+2 t_{i}}{3}}\left(x_{0}\right), \quad M_{j}=d_{j_{2}}^{\prod_{i=j_{2}}^{j-2} \frac{s_{i}+2 t_{i}}{3} t_{j-1}}\left(x_{0}\right),
$$

where $M_{j_{2}+1}=d_{j_{2}}^{t_{j_{2}}}\left(x_{0}\right)$. From (24), we have, for any $j \geq j_{2}+1$,

$$
\left(t_{j-1}-\frac{\beta}{3(j-1)}\right)\left(s_{j}+\frac{2 \beta}{3 j}\right)>t_{j-1} s_{j},
$$

thus

$$
d_{j_{2}}^{\prod_{i=j_{2}}^{j}} \frac{s_{i}+2 t_{i}}{3}\left(x_{0}\right)>d_{j_{2}}^{\prod_{i=j_{2}}^{j-2} \frac{s_{i}+2 t_{i}}{3} t_{j-1} s_{j}}\left(x_{0}\right)
$$

that is,

$$
L_{j+1}>M_{j}^{s_{j}}
$$


At the same time, it is evident that, for any $j \geq j_{2}+1$,

$$
M_{j+1}<L_{j}^{t_{j}} \text {. }
$$

Let

$$
\begin{aligned}
B_{\alpha}^{(6)}\left(j_{2}\right)=\left\{x \in(0,1]: d_{1}(x)=d_{1}\left(x_{0}\right), \ldots, d_{j_{2}}(x)=d_{j_{2}}\left(x_{0}\right)\right. \\
{\left.\left[L_{j+1}\right]<d_{j+1}(x) \leq\left[M_{j+1}\right] \text { for } j \geq j_{2}+1\right\} . }
\end{aligned}
$$

From (26), (27) and (23), we have

$$
B_{\alpha}^{(6)}\left(j_{2}\right) \subset B_{\alpha}^{(5)}\left(j_{2}\right) \subset B_{\alpha}^{(4)}\left(j_{2}\right) \subset B_{\alpha}^{(3)}\left(j_{2}\right) \subset B_{\alpha}^{(2)}\left(j_{1}\right) \subset B_{\alpha}^{(1)}\left(j_{0}\right) \subset B_{\alpha} .
$$

In the following, we find a lower bound of Hausdorff dimension of $B_{\alpha}^{(6)}\left(j_{2}\right)$ by using the mass distribution principle (Lemma 2.1 ).

First we introduce a kind of symbolic space defined in a similar way to the proof of Part I. For any $k \geq j_{2}$, let

$$
\begin{aligned}
D_{k}=\left\{\sigma=\left(\sigma_{1}, \ldots, \sigma_{k}\right)\right. & \in \mathbb{N}^{k}: \sigma_{j}=d_{j}\left(x_{0}\right) \text { for } 1 \leq j \leq j_{2}, \\
& \text { and } \left.\left[L_{j+1}\right]<\sigma_{j+1} \leq\left[M_{j+1}\right] \text { for } j_{2} \leq j<k\right\},
\end{aligned}
$$

and define

$$
D^{*}=\bigcup_{k=j_{2}}^{\infty} D_{k} .
$$

For any $k \geq j_{2}$ and $\sigma=\left(\sigma_{1}, \ldots, \sigma_{k}\right) \in D_{k}$, let $J_{\sigma}$ and $I_{\sigma}$ denote the following closed subintervals of $(0,1]$ :

$$
\begin{aligned}
& J_{\sigma}=\bigcup_{\left[L_{k+1}\right]<d \leq\left[M_{k+1}\right]} \operatorname{cl}\left\{x \in(0,1]: d_{1}(x)=\sigma_{1}, \ldots, d_{k}(x)=\sigma_{k}, d_{k+1}(x)=d\right\}, \\
& I_{\sigma}=\operatorname{cl}\left\{x \in(0,1]: d_{1}(x)=\sigma_{1}, \ldots, d_{k}(x)=\sigma_{k}\right\} ;
\end{aligned}
$$

each $J_{\sigma}$ is called an interval of $n$th order. Let

$$
E=\bigcap_{k=j_{2}}^{\infty} \bigcup_{\sigma \in D_{k}} J_{\sigma} .
$$

It is obvious that

$$
E=B_{\alpha}^{(6)}\left(j_{2}\right)
$$

From (10) and (11), we have

$$
\begin{aligned}
\left|I_{\sigma}\right| & =\frac{a_{1}\left(\sigma_{1}\right)}{b_{1}\left(\sigma_{1}\right)} \cdot \frac{a_{2}\left(\sigma_{2}\right)}{b_{2}\left(\sigma_{2}\right)} \cdots \frac{a_{k-1}\left(\sigma_{k-1}\right)}{b_{k-1}\left(\sigma_{k-1}\right)} \cdot \frac{1}{\left(\sigma_{k}-1\right) \sigma_{k}}, \\
\left|J_{\sigma}\right| & =\sum_{\left[L_{k+1}\right]<d \leq\left[M_{k+1}\right]} \frac{a_{1}\left(\sigma_{1}\right)}{b_{1}\left(\sigma_{1}\right)} \cdots \frac{a_{k}\left(\sigma_{k}\right)}{b_{k}\left(\sigma_{k}\right)} \cdot \frac{1}{(d-1) d} \\
& =\frac{a_{1}\left(\sigma_{1}\right)}{b_{1}\left(\sigma_{1}\right)} \cdots \frac{a_{k}\left(\sigma_{k}\right)}{b_{k}\left(\sigma_{k}\right)}\left(\frac{1}{\left[L_{k+1}\right]}-\frac{1}{\left[M_{k+1}\right]}\right) .
\end{aligned}
$$


Let $\mu$ be a probability mass distribution supported on $E$ such that for any $k \geq j_{2}$ and $\sigma \in D_{k}$,

$$
\mu\left(J_{\sigma}\right)=\frac{1}{\sharp D_{k}},
$$

where $\sharp$ denotes cardinality. We shall use $\mu$ to give a lower bound of the Hausdorff dimension of $E$.

For any $k \geq j_{2}$, write

$$
A_{k}=\sum_{j=j_{2}+1}^{k} \prod_{i=j_{2}}^{j-1} \frac{s_{i}+2 t_{i}}{3}, \quad B_{k}=\sum_{j=j_{2}+1}^{k+1} \prod_{i=j_{2}}^{j-2} \frac{s_{i}+2 t_{i}}{3} t_{j-1}, \quad C_{k}=\prod_{j=j_{2}}^{k} \frac{s_{i}+2 t_{i}}{3} .
$$

Then

$$
\begin{aligned}
& \lim _{k \rightarrow \infty} \frac{A_{k}}{B_{k}}=\lim _{k \rightarrow \infty} \frac{A_{k+1}-A_{k}}{B_{k+1}-B_{k}}=\frac{1}{\alpha \beta}, \\
& \lim _{k \rightarrow \infty} \frac{C_{k}}{B_{k}}=\lim _{k \rightarrow \infty} \frac{C_{k+1}-C_{k}}{B_{k+1}-B_{k}}=\frac{\alpha \beta-1}{\alpha \beta} .
\end{aligned}
$$

Now we estimate $\sharp D_{k}$. Notice that $x^{a}+1>(x+1)^{a}$ for any $0<a<1$ and $x>0$. By (22), for any $j \geq j_{2} \geq j_{0} \geq \beta / 3$ we have

$$
\left(\frac{1}{2} L_{j}\right)^{\beta / 3 j}>\frac{1}{2}\left(\left(L_{j}+1\right)^{\beta / 3 j}-1\right)>\frac{1}{2}\left(9^{\frac{3 j}{\beta} \cdot \frac{\beta}{3 j}}-1\right)=4 .
$$

Then for any $j \geq j_{2}+1$,

$$
\begin{aligned}
& {\left[M_{j}\right]-\left[L_{j}\right]>d_{j_{2}}^{\prod_{i=j_{2}}^{j-2} \frac{s_{i}+2 t_{i}}{3} t_{j-1}}-1-\left(1+\frac{1}{j^{2}}\right) d_{j_{2}}^{\prod_{i=j_{2}}^{j-1} \frac{s_{i}+2 t_{i}}{3}}}
\end{aligned}
$$

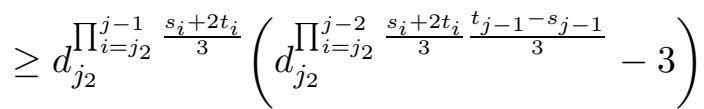

$$
\begin{aligned}
& \geq d_{j_{2}}^{\prod_{i=j_{2}}^{j-1} \frac{s_{i}+2 t_{i}}{3}}\left(\left(\frac{1}{2} L_{j-1}\right)^{\frac{\beta}{3(j-1)}}-3\right) \\
& \geq d_{j_{2}}^{\prod_{i=j_{2}}^{j-1} \frac{s_{i}+2 t_{i}}{3}}=d_{j_{2}}^{C_{j-1}} \text {. }
\end{aligned}
$$

Thus for any $k \geq j_{2}+1$,

$$
\sharp D_{k}=\prod_{j=j_{2}+1}^{k}\left(\left[M_{j}\right]-\left[L_{j}\right]\right) \geq d_{j_{2}}^{\sum_{j=j_{2}+1}^{k} \prod_{i=j_{2}}^{j-1} \frac{s_{i}+2 t_{i}}{3}}=d_{j_{2}}^{A_{k}} .
$$

For any $s$ satisfying

$$
0<s<\frac{1}{\alpha \beta\left(2-\left(\alpha+\frac{2}{j_{2}}\right)^{-1}\right)-(\alpha \beta-1)},
$$


there exists $1 / 2>\eta_{0}>0$ such that for any $0<\eta<\eta_{0}$,

$$
s<\frac{1-\eta}{\alpha \beta\left(2-\left(\alpha+\frac{2}{j_{2}}\right)^{-1}\right)-(\alpha \beta-1-\eta)} .
$$

For any fixed $0<\eta<\eta_{0}$, by (32) and (33), there exists $k_{0}(\eta)$ such that for any $k \geq k_{0}(\eta)$,

$$
\frac{A_{k}}{B_{k}}>\frac{1-\eta}{\alpha \beta}, \quad \frac{C_{k}}{B_{k}}>\frac{\alpha \beta-1-\eta}{\alpha \beta} .
$$

For any $x \in E$, we prove that

$$
\liminf _{r \rightarrow 0} \frac{\log \mu(B(x, r))}{\log r} \geq s
$$

For such $x$, there exists $\sigma=\left(\sigma_{1}, \sigma_{2}, \ldots\right)$ such that $\sigma_{i}=d_{i}\left(x_{0}\right)$ for $1 \leq i \leq j_{2}$, and for any $k \geq j_{2},(\sigma \mid k):=\left(\sigma_{1}, \ldots, \sigma_{k}\right) \in D_{k}$ and $d_{j}(x)=\sigma_{j}$ for any $j \geq 1$. Thus $x \in J_{\sigma_{1} \cdots \sigma_{k}}$ for any $k \geq j_{2}$.

From the proof of Theorem 6.1 in [5], we know that, for any $k \geq j_{2}$, the right endpoint of the interval $J_{\sigma_{1} \cdots \sigma_{k}}$, i.e., $\max \left\{y \in(0,1]: y \in J_{\sigma_{1} \cdots \sigma_{k}}\right\}$, is

$$
\frac{1}{\sigma_{1}}+\frac{a_{1}\left(\sigma_{1}\right)}{b_{1}\left(\sigma_{1}\right)} \cdot \frac{1}{\sigma_{2}}+\cdots+\frac{a_{1}\left(\sigma_{1}\right)}{b_{1}\left(\sigma_{1}\right)} \cdots \frac{a_{k}\left(\sigma_{k}\right)}{b_{k}\left(\sigma_{k}\right)} \cdot \frac{1}{\left[L_{k+1}\right]},
$$

and the left endpoint, i.e., $\min \left\{y \in(0,1]: y \in J_{\sigma_{1} \cdots \sigma_{k}}\right\}$, is

$$
\frac{1}{\sigma_{1}}+\frac{a_{1}\left(\sigma_{1}\right)}{b_{1}\left(\sigma_{1}\right)} \cdot \frac{1}{\sigma_{2}}+\cdots+\frac{a_{1}\left(\sigma_{1}\right)}{b_{1}\left(\sigma_{1}\right)} \cdots \frac{a_{k}\left(\sigma_{k}\right)}{b_{k}\left(\sigma_{k}\right)} \cdot \frac{1}{\left[M_{k+1}\right]}
$$

(i) If $\sigma_{k}-1>\left[L_{k}\right]$, from the definition of $h_{j}(d),(38)$ and (39), we know that the gap between $J_{\sigma_{1} \cdots \sigma_{k}}$ and $J_{\sigma_{1} \cdots \sigma_{k}-1}$, denoted by $g_{k}^{r}(x)$, is

$$
\begin{gathered}
\frac{a_{1}\left(\sigma_{1}\right)}{b_{1}\left(\sigma_{1}\right)} \cdots \frac{a_{k-1}\left(\sigma_{k-1}\right)}{b_{k-1}\left(\sigma_{k-1}\right)} \cdot \frac{a_{k}\left(\sigma_{k}-1\right)}{b_{k}\left(\sigma_{k}-1\right)} \cdot \frac{1}{\left[M_{k+1}\right]} \\
\quad+\frac{a_{1}\left(\sigma_{1}\right)}{b_{1}\left(\sigma_{1}\right)} \cdots \frac{a_{k}\left(\sigma_{k}\right)}{b_{k}\left(\sigma_{k}\right)}\left(\frac{1}{h_{k}\left(\sigma_{k}\right)}-\frac{1}{\left[L_{k+1}\right]}\right) \\
\geq \frac{a_{1}\left(\sigma_{1}\right)}{b_{1}\left(\sigma_{1}\right)} \cdots \frac{a_{k-1}\left(\sigma_{k-1}\right)}{b_{k-1}\left(\sigma_{k-1}\right)} \cdot \frac{a_{k}\left(\sigma_{k}-1\right)}{b_{k}\left(\sigma_{k}-1\right)} \cdot \frac{1}{\left[M_{k+1}\right]}=: G_{k}^{r}(x) .
\end{gathered}
$$

(ii) Suppose $\sigma_{k}=\left[L_{k}\right]+1$. If $\sigma_{j}=\left[L_{j}\right]+1$ for any $j_{2}+1 \leq j \leq k$, let $g_{k}^{r}(x)=g_{k-1}^{r}(x)=\cdots=g_{j_{2}+1}^{r}(x)=\infty$ and $G_{k}^{r}(x)=G_{k-1}^{r}(x)=\cdots=$ $G_{j_{2}+1}^{r}(x)=\infty$. If there exists $j_{2}+1 \leq j \leq k$ such that $\sigma_{j}>\left[L_{j}\right]+1$, let $\widetilde{j}=\max \left\{j: j_{2}+1 \leq j \leq k, \sigma_{j}>\left[L_{j}\right]+1\right\}$. Define $g_{k}^{r}(x)=g_{k-1}^{r}(x)=\cdots=$ $g_{\tilde{j}}^{r}(x)$ and $G_{k}^{r}(x)=G_{k-1}^{r}(x)=\cdots=G_{\tilde{j}}^{r}(x)$. 
(iii) If $\sigma_{k}+1 \leq\left[M_{k}\right]$, the gap between $J_{\sigma_{1} \cdots \sigma_{k}}$ and $J_{\sigma_{1} \cdots \sigma_{k}+1}$, denoted by $g_{k}^{l}(x)$, satisfies

$$
g_{k}^{l}(x) \geq \frac{a_{1}\left(\sigma_{1}\right)}{b_{1}\left(\sigma_{1}\right)} \cdots \frac{a_{k}\left(\sigma_{k}\right)}{b_{k}\left(\sigma_{k}\right)} \cdot \frac{1}{\left[M_{k+1}\right]}=: G_{k}^{l}(x) .
$$

(iv) If $\sigma_{k}=\left[M_{k}\right]$, let $g_{k}^{l}(x)$ denote the distance between the left endpoint of $J_{\sigma_{1} \cdots \sigma_{k-1}}$ and the left endpoint of $J_{\sigma_{1} \cdots \sigma_{k}}$. Then

$$
g_{k}^{l}(x)=\frac{a_{1}\left(\sigma_{1}\right)}{b_{1}\left(\sigma_{1}\right)} \cdots \frac{a_{k}\left(\sigma_{k}\right)}{b_{k}\left(\sigma_{k}\right)} \cdot \frac{1}{\left[M_{k+1}\right]}=: G_{k}^{l}(x) .
$$

Define

$$
G_{k}(x):=\min \left\{G_{k}^{l}(x), G_{k}^{r}(x)\right\} .
$$

Let $k>\max \left(1 / 3(\alpha-1), j_{2}\right)$. By $(24)$, we have $\alpha \beta / 3 k<(\alpha-1) t_{k}$, which implies $t_{k} \beta<\left(t_{k}-\beta / 3 k\right) \alpha \beta$. Thus $t_{k} \beta<\left(\left(s_{k}+2 t_{k}\right) / 3\right) t_{k+1}$, so

$$
M_{k+1}^{\beta}<M_{k+2} \text {. }
$$

On the other hand, by (17), we have

$$
\sigma_{k+1}-1 \geq d_{j_{2}}\left(x_{0}\right)-1=d_{j_{2}}-1>c^{3} \cdot 2^{\beta+2} .
$$

Combining (40) and (41), we have

$$
c^{3} \cdot 2^{\beta+2} \frac{\sigma_{k+1}^{\beta}}{\sigma_{k+1}\left(\sigma_{k+1}-1\right)} M_{k+1}<M_{k+2} .
$$

By the definition of $h_{j}(d)$, and since $h_{j}(d)$ is of order $\beta$, we have

$$
\frac{h_{k}\left(\sigma_{k}\right)}{\sigma_{k}\left(\sigma_{k}-1\right)} \cdot \frac{h_{k+1}\left(\sigma_{k+1}-1\right)}{\left(\sigma_{k+1}-1\right)\left(\sigma_{k+1}-2\right)} \cdot \frac{1}{\left[M_{k+2}\right]}<\frac{h_{k}\left(\sigma_{k}-1\right)}{\left(\sigma_{k}-1\right)\left(\sigma_{k}-2\right)} \cdot \frac{1}{\left[M_{k+1}\right]},
$$

i.e.

$$
\begin{aligned}
& \frac{a_{1}\left(\sigma_{1}\right)}{b_{1}\left(\sigma_{1}\right)} \cdots \frac{a_{k+1}\left(\sigma_{k+1}-1\right)}{b_{k+1}\left(\sigma_{k+1}-1\right)} \cdot \frac{1}{\left[M_{k+2}\right]} \\
& \quad<\frac{a_{1}\left(\sigma_{1}\right)}{b_{1}\left(\sigma_{1}\right)} \cdots \frac{a_{k-1}\left(\sigma_{k-1}\right)}{b_{k-1}\left(\sigma_{k-1}\right)} \cdot \frac{a_{k}\left(\sigma_{k}-1\right)}{b_{k}\left(\sigma_{k}-1\right)} \cdot \frac{1}{\left[M_{k+1}\right]},
\end{aligned}
$$

that is, $G_{k+1}^{r}(x)<G_{k}^{r}(x)$. In the same way, we have $G_{k+1}^{l}(x)<G_{k}^{l}(x)$. So $k>\max \left(1 / 3(\alpha-1), j_{2}\right)$ implies $G_{k+1}(x)<G_{k}(x)$.

Let $K_{1}=\max \left(1 / 3(\alpha-1), j_{2}\right)$. For any $0<r<\min _{j_{2}<j \leq K_{1}}\left\{G_{j}(x)\right\}$, there exists $k \geq K_{1}$ such that $G_{k+1}(x) \leq r<G_{k}(x)$. Thus $B(x, r)$ can intersect only one $k$ th-order interval, which is $J_{\sigma_{1} \cdots \sigma_{k}}$. Now we find an upper bound of the number of $(k+1)$ th-order intervals, the $(k+1)$ th-order subintervals of $J_{\sigma_{1} \cdots \sigma_{k}}$, which intersect $B(x, r)$. Since $J_{\sigma} \subset I_{\sigma}$, we only need to consider the number of $\left\{I\left(\sigma_{1}, \ldots, \sigma_{k}, j\right)\right\}_{\left[L_{k+1}\right]<j \leq\left[M_{k+1}\right]}$ which intersect $B(x, r)$. By (29), the definition of $h_{j}(d)$ and the fact that $E=B_{\alpha}^{(6)}\left(j_{2}\right) \subset B_{\alpha}^{(1)}\left(j_{0}\right)$, 
we have

$$
\begin{aligned}
& \left|I_{\sigma_{1} \cdots \sigma_{k} j}\right|=\frac{a_{1}\left(\sigma_{1}\right)}{b_{1}\left(\sigma_{1}\right)} \cdots \frac{a_{k}\left(\sigma_{k}\right)}{b_{k}\left(\sigma_{k}\right)} \cdot \frac{1}{j(j-1)} \\
& \quad=\prod_{l=1}^{j_{2}-1} \frac{a_{l}\left(\sigma_{l}\right)}{b_{l}\left(\sigma_{l}\right)} \cdot \frac{1}{\sigma_{j_{2}}\left(\sigma_{j_{2}}-1\right)} \cdot \frac{h_{j_{2}}\left(\sigma_{j_{2}}\right)}{\sigma_{j_{2}+1}\left(\sigma_{j_{2}+1}-1\right)} \cdots \frac{h_{k-1}\left(\sigma_{k-1}\right)}{\sigma_{k}\left(\sigma_{k}-1\right)} \cdot \frac{h_{k}\left(\sigma_{k}\right)}{j(j-1)} \\
& \quad \geq c\left(j_{2}\right) \frac{\sigma_{j_{2}+1}^{\left(\alpha+2 / j_{2}\right)^{-1}}}{\sigma_{j_{2}+1}^{2}} \cdots \frac{\sigma_{k}^{(\alpha+2 /(k-1))^{-1}}}{\sigma_{k}^{2}} \cdot \frac{j^{(\alpha+2 / k)^{-1}}}{j^{2}} \\
& \quad \geq c\left(j_{2}\right) \frac{1}{\left(\sigma_{j_{2}+1} \cdots \sigma_{k} \cdot j\right)^{2-\left(\alpha+2 / j_{2}\right)^{-1}}} \\
& \quad \geq c\left(j_{2}\right) \frac{1}{\left(M_{j_{2}+1} \cdots M_{k+1}\right)^{2-\left(\alpha+2 / j_{2}\right)^{-1}}}=c\left(j_{2}\right) d_{j_{2}}^{-\left(2-\left(\alpha+2 / j_{2}\right)^{-1}\right) B_{k}},
\end{aligned}
$$

where

$$
c\left(j_{2}\right)=\prod_{l=1}^{j_{2}-1} \frac{a_{l}\left(\sigma_{l}\right)}{b_{l}\left(\sigma_{l}\right)} \cdot \frac{1}{\sigma_{j_{2}}\left(\sigma_{j_{2}}-1\right)}=\prod_{l=1}^{j_{2}-1} \frac{a_{l}\left(d_{l}\left(x_{0}\right)\right)}{b_{l}\left(d_{l}\left(x_{0}\right)\right)} \cdot \frac{1}{d_{j_{2}}\left(x_{0}\right)\left(d_{j_{2}}\left(x_{0}\right)-1\right)},
$$

which does not depend on $x$. So the number of $(k+1)$ th-order intervals which intersect $B(x, r)$ is not more than

$$
4 r\left(c\left(j_{2}\right) d_{j_{2}}^{-\left(2-\left(\alpha+2 / j_{2}\right)^{-1}\right) B_{k}}\right)^{-1}
$$

By (31), (34)-(37), (42), we have

$$
\begin{aligned}
\mu(B(x, r)) & \leq \frac{1}{\sharp D_{k+1}} \min \left\{\frac{4 r}{\left.c\left(j_{2}\right) d_{j_{2}}^{-\left(2-\left(\alpha+2 / j_{2}\right)^{-1}\right) B_{k}},\left[M_{k+1}\right]-\left[L_{k+1}\right]\right\}}\right. \\
& \leq \frac{1}{\sharp D_{k+1}}\left(\frac{4 r}{c\left(j_{2}\right) d_{j_{2}}^{-\left(2-\left(\alpha+2 / j_{2}\right)^{-1}\right) B_{k}}}\right)^{s}\left(\left[M_{k+1}\right]-\left[L_{k+1}\right]\right)^{1-s} \\
& \leq \frac{1}{\sharp D_{k}}\left(\frac{4 r}{c\left(j_{2}\right)} d_{j_{2}}^{\left(2-\left(\alpha+2 / j_{2}\right)^{-1}\right) B_{k}} \cdot \frac{1}{\left[M_{k+1}\right]-\left[L_{k+1}\right]}\right)^{s} \\
& \leq d_{j_{2}}^{-A_{k}}\left(\frac{4 r}{c\left(j_{2}\right)}\right)^{s} d_{j_{2}}^{\left(2-\left(\alpha+2 / j_{2}\right)^{-1}\right) B_{k} s} d_{j_{2}}^{-C_{k} s} \\
& \leq\left(\frac{4 r}{c\left(j_{2}\right)}\right)^{s} d_{j_{2}}^{B_{k}\left(-\frac{1-\eta}{\alpha \beta}+s\left(2-\left(\alpha+2 / j_{2}\right)^{-1}\right)-s \frac{\alpha \beta-1-\eta}{\alpha \beta}\right)} \\
& \leq\left(\frac{4 r}{c\left(j_{2}\right)}\right)^{s}=\left(\frac{4}{c\left(j_{2}\right)}\right)^{s} r^{s} .
\end{aligned}
$$

By Lemma 2.1, we have

$$
\operatorname{dim}_{\mathrm{H}} B^{(6)}\left(j_{2}\right)=\operatorname{dim}_{\mathrm{H}} E \geq s .
$$


Therefore,

$$
\begin{aligned}
\operatorname{dim}_{\mathrm{H}} B^{(1)}\left(j_{0}\right) \geq \operatorname{dim}_{\mathrm{H}} B^{(6)}\left(j_{2}\right) & \geq \frac{1}{\alpha \beta\left(2-\left(\alpha+2 / j_{2}\right)^{-1}\right)-(\alpha \beta-1)} \\
& \geq \frac{1}{\alpha \beta\left(2-\left(\alpha+2 / j_{0}\right)^{-1}\right)-(\alpha \beta-1)} .
\end{aligned}
$$

Thus

$$
\operatorname{dim}_{\mathrm{H}} B_{\alpha} \geq \frac{1}{\alpha \beta\left(2-\left(\alpha+2 / j_{0}\right)^{-1}\right)-(\alpha \beta-1)}
$$

for any $j_{0}>\beta / 3, j_{0} \in \mathbb{N}$, which implies

$$
\operatorname{dim}_{\mathrm{H}} B_{\alpha} \geq \frac{1}{(\alpha-1) \beta+1} \text {. }
$$

This completes the proof of Theorem 2.3.

We now list some special cases which satisfy the assumptions of Theorem 2.3.

EXAMPLE 1 (Engel expansion). Let $a_{n}\left(d_{n}\right)=1, b_{n}\left(d_{n}\right)=d_{n}(n=$ $1,2, \ldots)$. Then $(2)$, together with the algorithm (1), yields the Engel expansion of $x$,

$$
x=\frac{1}{d_{1}(x)}+\frac{1}{d_{1}(x) d_{2}(x)}+\cdots+\frac{1}{d_{1}(x) d_{2}(x) \cdots d_{n}(x)}+\cdots .
$$

In this case, $h_{n}(j)=j-1$ is of order 1 . By Theorem 2.3, we have

Corollary 2.4. For the Engel expansion,

$$
\operatorname{dim}_{\mathrm{H}}\left\{x \in(0,1]: \lim _{n \rightarrow \infty} \frac{\log d_{n+1}(x)}{\log d_{n}(x)}=\alpha\right\}=\frac{1}{\alpha} \quad \text { for any } \alpha \geq 1 .
$$

ExAmple 2 (Sylvester expansion). Choose $a_{n}\left(d_{n}\right)=1, b_{n}\left(d_{n}\right)=1$ $(n=1,2, \ldots)$. We get the Sylvester expansion of $x$,

$$
x=\frac{1}{d_{1}(x)}+\frac{1}{d_{2}(x)}+\cdots+\frac{1}{d_{n}(x)}+\cdots .
$$

Here $h_{n}(j)=j(j-1)$ is of order 2. By Theorem 2.3, we have

Corollary 2.5. For the Sylvester expansion,

$$
\operatorname{dim}_{\mathrm{H}}\left\{x \in(0,1]: \lim _{n \rightarrow \infty} \frac{\log d_{n+1}(x)}{\log d_{n}(x)}=\alpha\right\}=\frac{1}{\alpha-1} \quad \text { for any } \alpha \geq 2 \text {. }
$$

EXAmPle 3 (Cantor product). Take $a_{n}\left(d_{n}\right)=d_{n}+1 b_{n}\left(d_{n}\right)=d_{n}$ $(n=1,2, \ldots)$. The expansion (2) yields the Cantor product

$$
1+x=\left(1+\frac{1}{d_{1}(x)}\right)\left(1+\frac{1}{d_{2}(x)}\right) \cdots\left(1+\frac{1}{d_{n}(x)}\right) \cdots .
$$

Here $h_{n}(j)=j^{2}-1$ is of order 2. By Theorem 2.3, we have 
COROLlary 2.6. For the Cantor product, $\operatorname{dim}_{\mathrm{H}}\left\{x \in(0,1]: \lim _{n \rightarrow \infty} \frac{\log d_{n+1}(x)}{\log d_{n}(x)}=\alpha\right\}=\frac{1}{\alpha-1} \quad$ for any $\alpha \geq 2$.

EXAMPLE 4 (Modified Engel expansion). Let $a_{n}\left(d_{n}\right)=1, b_{n}\left(d_{n}\right)=d_{n}-1$ $(n=1,2, \ldots)$. We get the modified Engel expansion of $x$,

$$
x=\frac{1}{d_{1}(x)}+\cdots+\frac{1}{\left(d_{1}(x)-1\right)\left(d_{2}(x)-1\right) \cdots\left(d_{n-1}(x)-1\right) d_{n}(x)}+\cdots .
$$

Thus $h_{n}(j)=j$ is of order 1 . By Theorem 2.3, we have

Corollary 2.7. For the modified Engel expansion,

$$
\operatorname{dim}_{\mathrm{H}}\left\{x \in(0,1]: \lim _{n \rightarrow \infty} \frac{\log d_{n+1}(x)}{\log d_{n}(x)}=\alpha\right\}=\frac{1}{\alpha} \quad \text { for any } \alpha \geq 1 .
$$

ExAmple 5 (Daróczy-Kátai-Birthday expansion). Choose $a_{n}\left(d_{n}\right)=d_{n}$, $b_{n}\left(d_{n}\right)=1(n=1,2, \ldots)$. The resulting series expansion of $x$ takes the form

$$
x=\frac{1}{d_{1}(x)}+\frac{d_{1}(x)}{d_{2}(x)}+\cdots+\frac{d_{1}(x) d_{2}(x) \cdots d_{n-1}(x)}{d_{n}(x)}+\cdots .
$$

This Daróczy-Kátai-Birthday expansion was introduced for the first time in Galambos [6]. Here $h_{n}(j)=j^{2}(j-1)$ is of order 3. By Theorem 2.3, we have

Corollary 2.8. For the Daróczy-Kátai-Birthday expansion, $\operatorname{dim}_{\mathrm{H}}\left\{x \in(0,1]: \lim _{n \rightarrow \infty} \frac{\log d_{n+1}(x)}{\log d_{n}(x)}=\alpha\right\}=\frac{1}{\alpha-2} \quad$ for any $\alpha \geq 3$.

Acknowledgements. This work was partially done during the second author's visit to the LAMFA, CNRS UMR 6140, Amiens. He would like to thank that institution for warm hospitality. This work was supported by the Kua-Shi-Ji foundation of Educational Committee and NSFC (10571138).

\section{References}

[1] K. J. Falconer, Techniques in Fractal Geometry, Wiley, 1997.

[2] J. Galambos, The ergodic properties of the denominators in the Oppenheim expansion of real numbers into infinite series of rationals, Quart. J. Math. Oxford Ser. (2) 21 (1970), 177-191.

[3] -, Further ergodic results on the Oppenheim series, ibid. 25 (1974), 135-141.

[4] - On the speed of the convergence of the Oppenheim series, Acta Arith. 19 (1971), 335-342.

[5] - Reprentations of Real Numbers by Infinite Series, Lecture Notes in Math. 502, Springer, 1976.

[6] - Further metric results on series expansions, Publ. Math. Debrecen 52 (1998), 377-384. 
[7] A. Oppenheim, The representation of real numbers by infinite series of rationals, Acta Arith. 21 (1972), 391-398.

[8] F. Schweiger, Ergodic Theory of Fibred Systems and Metric Number Theory, Clarendon Press, Oxford, 1995.

[9] W. Vervaat, Success Epochs in Bernoulli Trials, Math. Center Tracts 42, Math. Centrum, Amsterdam, 1972.

[10] B. W. Wang and J. Wu, The Oppenheim series expansions and a problem of Galambos, Publ. Math. Debrecen, to appear.

[11] J. Wu, The Oppenheim series expansions and Hausdorff dimensions, Acta Arith. 107 (2003), 345-355.

[12] - , How many points have the same Engel and Sylvester expansions?, J. Number Theory 103 (2003), 16-26.

Bao-Wei Wang

Department of Mathematics

Wuhan University

Wuhan, Hubei, 430072, P.R. China

E-mail: bwei_wang@yahoo.com.cn
Jun $\mathrm{Wu}$

Department of Mathematics Huazhong University of Science and Technology Wuhan, Hubei, 430074, P.R. China E-mail: wujunyu@public.wh.hb.cn

Received on 20.6.2005

and in revised form on 25.9.2005 